\title{
FOTODEGRADACIÓN HETEROGÉNEA DE BISFENOL A EN AGUA CON DIÓXIDO DE TITANIO
}

\author{
Luisa F. Gómez y Victor M. Sarria* \\ Departamento de Química, Universidad de los Andes, Carrera 1E No 18ª -10, Bogotá, Colombia
}

Recebido em 14/5/08; aceito em 5/12/08; publicado na web em 11/5/09

\begin{abstract}
HETEROGENEOUS PHOTODEGRADATION OF BISPHENOL A IN WATER WITH TITANIUM DIOXIDE. Bisphenol A (BPA) is a monomer used in epoxy resin and polycarbonate manufacture. This molecule is considered as an endocrine disruptor that causes different diseases. The human exposition to this non biodegrable substance is increasing in the time; in particular, water is contaminated by industrial remainder flow. In this article heterogeneous photo degradation of a solution of BPA in water solution using a catalytic photo reactor with UV light and titanium dioxide $\left(\mathrm{TiO}_{2}\right)$ was evaluated. High performance liquid chromatography (HPLC) was used to analyze the photo degradation of BPA solutions. The influence of titanium dioxide amount, BPA concentration, reaction temperature and the catalyst state like suspension and immobilized were also determinated. The highest elimination of BPA was $83.2 \%$, in $240 \mathrm{~min}$, beginning with $0.05 \mathrm{mM}$ of $\mathrm{BPA}$ and $100 \mathrm{mg} / \mathrm{L}$ of $\mathrm{TiO}_{2}$ in suspension.
\end{abstract}

Keywords: photo degradation; $\mathrm{TiO}_{2}$; Bisphenol A.

\section{INTRODUCCION}

Actualmente se estima que en promedio 2.000 sustancias químicas, se incorporan anualmente al medio ambiente y a la vida humana. El hombre y su entorno resultan expuestos a estos compuestos desde el momento de su fabricación hasta su distribución, uso y posible degradación. La literatura científica especializada relaciona este crecimiento tan acelerado, como causa de nuevos síndromes y desarrollo de nuevas enfermedades. ${ }^{1}$

Se ha presumido recientemente que ciertos productos químicos denominados como disruptores endocrinos tienen la capacidad de alterar el equilibrio hormonal y causar efectos adversos para la salud de los seres humanos y de la fauna, a concentraciones muy bajas. ${ }^{1-3}$

La exposición de los seres vivos a estas sustancias es cada vez mayor, ya que son ampliamente utilizados, poseen baja degradabilidad, son transportados por aire, suelo y agua, además pueden bioacumularse en la cadena trófica debido a que se almacenan en la grasa de los organismos y son transmitidos a la descendencia a través de la madre durante la gestación y después de la lactancia., ${ }^{1,4}$

El Bisfenol A (BPA) fue catalogado como disruptor endocrino según el ministerio del medio ambiente en Japón. ${ }^{5}$ Esta sustancia es un monómero ampliamente utilizado en la industria de polímeros, resinas epóxicas y policarbonatos. Estudios atribuyen a esta sustancia; poliquistesis ovárica, problemas de fertilidad femenina, cromosomas fetales anormales, feminización, alteraciones reproductivas inmunológicas y de desarrollo. ${ }^{5,6}$

Según la literatura se ha encontrado trazas de Bisfenol A en aire $\left(2-208 \mathrm{ng} / \mathrm{m}^{3}\right)$, suelo y agua $(8-21 \mathrm{ng} / \mathrm{ml}) .{ }^{7}$ Se ha demostrado que esta sustancia contenida en resinas epóxicas como los productos enlatados alimenticios, puede migrar desde las paredes del material hasta el interior del producto. Se ha identificado trazas de BPA en animales marinos disponibles para el consumo humano. ${ }^{7}$ Debido a las anteriores evidencias, varios países han restringido la concentración permisible del BPA. En 1998, la EPA consideró que a partir de una dosis diaria igual o mayor a $0.05 \mathrm{mg} / \mathrm{kg}$ peso cuerpo el BPA podría generar consecuencias negativas, ${ }^{6}$ sin embargo en el 2000 Estados Unidos consideró que este valor debería ser inferior a $10 \mu \mathrm{g} / \mathrm{kg}$ peso cuerpo (BW). ${ }^{6}$ Con respecto a la migración

\footnotetext{
*e-mail: vsarria@uniandes.edu.co
}

del BPA desde un determinado material, Japón estableció una restricción de $2.5 \mathrm{mg} / \mathrm{kg},{ }^{6}$ y en 2004 Estados Unidos $0,6 \mathrm{mg} / \mathrm{kg}$ para la migración. ${ }^{6}$ Es importante considerar que actualmente en Colombia no existe ningún tipo de restricción respecto al manejo, uso y descarga del BPA.

Teniendo en cuenta que el agua es uno de los principales medios donde se deposita este compuesto como residuo de procesos industriales y de desecho, se intenta crear sistemas para tratamientos de agua y purificación de la misma. Sin embargo, las tecnologías tradicionales que se utilizan para la separación de sustancias orgánicas del agua tratada están basadas en procesos de adsorción con carbón activado o por arrastre con aire. Esto implica que dichos procesos sólo transfieren los contaminantes de su fase acuosa a otra que resulta también contaminada, por lo tanto el problema persiste. Este problema ha generado la necesidad de implementar nuevos procesos para tratar compuestos no biodegradables.

Actualmente, existe un grupo de tecnologías basadas en procesos de destrucción de los contaminantes por medio de radicales hidroxilos $\left({ }^{\circ} \mathrm{OH}\right)$, las cuales poseen un alto potencial de oxidación y atribuyen la capacidad de oxidar o degradar casi la totalidad del contaminante transformándolo en compuestos inofensivos al medio ambiente e incluso puede llegar hasta la mineralización o formación de $\mathrm{CO}_{2}$. Estas tecnologías son llamadas "procesos avanzados de oxidación” (POAs), las cuales están comenzando a ser implementadas en América del Norte, Europa y Japón. La importancia de estos procesos y el interés de estudiarlos, se debe a que la energía solar puede ser utilizada como fuente de fotones generando un ahorro energético. ${ }^{8}$ Los POAs abarcan procesos como ozono/luz $\mathrm{UV}, \mathrm{H}_{2} \mathrm{O}_{2} / \mathrm{luz} \mathrm{UV}$, ultrasonido, foto-catálisis heterogénea y homogénea, y tratamientos electroquímicos. ${ }^{8}$

La foto-catálisis heterogénea sobre dióxido de titanio $\left(\mathrm{TiO}_{2}\right)$ es uno de los procesos más estudiados. $\mathrm{El} \mathrm{TiO}_{2}$ es un semiconductor que cumple la función de catalizador, por lo tanto aumenta la velocidad de reacción sin alterar el equilibrio. Este se activa con la luz ultravioleta y produce radicales $\left({ }^{\circ} \mathrm{OH}\right)$, los cuales oxidan la materia orgánica del contaminante. La remoción del contaminante ocurre cuando el catalizador $\left(\mathrm{TiO}_{2}\right)$ es irradiado por la luz UV. Esta fuente de energía excita a un electrón promoviéndolo desde la banda de valencia (baja energía) hacia la banda de conducción (alta energía), dejando un hueco positivo en la primera banda. El mecanismo se muestra a continuación: 


$$
\mathrm{TiO}_{2}\left(h^{+}\right)+\mathrm{HO}^{-} \rightarrow \mathrm{TiO}_{2}+{ }^{\bullet} \mathrm{OH}
$$

Este hueco al entrar en contacto con el agua $\left(\mathrm{H}_{2} \mathrm{O}\right)$ o con un ion $\mathrm{OH}-$, produce los radicales hidroxilo $\left({ }^{\circ} \mathrm{OH}\right)$.

$$
\begin{aligned}
& \mathrm{TiO}_{2}\left(h^{+}\right)+\mathrm{H}_{2} \mathrm{O} \rightarrow \mathrm{TiO}_{2}+{ }^{\circ} \mathrm{OH}+\mathrm{H}^{+} \\
& \mathrm{TiO}_{2}\left(h^{+}\right)+\mathrm{HO}^{-} \rightarrow \mathrm{TiO}_{2}+{ }^{\bullet} \mathrm{OH}
\end{aligned}
$$

Las especies formadas, logran modificar químicamente a la sustancia toxica convirtiéndola en sustancias intermediarias inofensivas y biodegradables para el medio ambiente. ${ }^{9}$

En los últimos años se han desarrollando procesos para degradar disruptores endocrinos a través de POA's. El BPA fue degradado por medio de foto-catálisis con $\mathrm{TiO}_{2}$ en china en el $2005,{ }^{6}$ la foto degradación con $\mathrm{UV} / \mathrm{H}_{2} \mathrm{O}_{2}$ de estradiol (E2), etenil estradiol, nonifenol (NP), Bisfenol A (BPA), se evaluó en Estados Unidos en el 2006. ${ }^{10}$ La degradación de Bisfenol A (BPA) por medio de foto-catálisis heterogénea con zeolitas en dióxido de titanio en Japón, 2003. ${ }^{11}$ En Japón en el 2002, se estudio el mecanismo de foto degradación de Bisfenol A en superficies con dióxido de titanio y agua. ${ }^{5}$

En este trabajo se evalúa los procesos de oxidación avanzada como alternativa de foto degradación de Bisfenol A en agua con dióxido de titanio. Se determina la concentración adecuada de catalizador, la dependencia de la temperatura de reacción en la remoción de contaminante, y se inmoviliza el dióxido de titanio en láminas de acrílico, acetato y vidrio.

\section{PARTE EXPERIMENTAL}

\section{Materiales y reactivos}

Los materiales, equipos y reactivos utilizados en el análisis fueron: Bisfenol A: 4,4'-isopropilidendifenol, Aldrich, Estado solidó; dióxido de titanio $\left(\mathrm{TiO}_{2}\right.$ ): Degussa P25, densidad $3.8 \mathrm{gr} / \mathrm{cm}^{3}$, área superficie BET $55 \mathrm{~m}^{2} / \mathrm{mg}$, tamaño de partícula promedio $30 \mathrm{~mm}$, diámetro de poro $5.6 \mathrm{~mm}$ (Mesoporo) ${ }^{20}$; foto-reactor catalítico: es un circuito que consta de una superficie tubular concéntrica conformada por una lámpara (Lexmana) de radiación UV de $40 \mathrm{~W}$ de potencia y emisión de $360 \mathrm{~nm}$ (longitud 124 y diámetro $2 \mathrm{~cm}$ ) y un tubo Pirex que rodea la fuente de radiación (diámetro 4 y longitud $110 \mathrm{~cm}$ ). El espacio vació entre estos dos equipos corresponde al área por donde fluye la solución. La recirculación de esta solución se lleva a cabo mediante mangueras de caucho siliconado $(0.5 \mathrm{~cm}$ de diámetro $)$ conectadas a una bomba peristáltica que se encarga de transportar la solución a un recipiente de reciclo (Beaker de 1 L). Para generar una mezcla homogénea y un mezclado continuo se utiliza un agitador magnético y una plancha de agitación. El flujo empleado fue de 400 $\mathrm{mL} / \mathrm{min}$, el cual produce un numero de Reynolds en la manguera de 2766.72 caracterizando un flujo de transición entre laminar y turbulento, con el fin de favorecer la transferencia de masa y evitar la sedimentación del catalizador.

\section{Metodología y métodos de análisis}

El procedimiento experimental se divide en dos partes: usando el catalizador en suspensión y fijo.

\section{Procedimiento experimental $\mathrm{TiO}_{2}$ en suspensión}

Se prepararon cuatro concentraciones diferentes de Bisfenol A: $0 ; 0.01 ; 0.05 ; 0.1 \mathrm{mM}$ en $1 \mathrm{~L}$ de agua. Se utilizaron cuatro cantidades diferentes de dióxido de titanio 0; 100; 250; $500 \mathrm{mg} / \mathrm{L} \mathrm{de} \mathrm{TiO}_{2}$.
La solución agua- dióxido de titanio- Bisfenol A, fue agitada durante aproximadamente $10 \mathrm{~min}$ con un agitador magnético y una plancha de agitación, antes de ser recirculada por el reactor foto catalítico. Cada prueba fue de $4 \mathrm{~h}$ y se tomaron muestras cada hora. Estas fueron filtradas con un filtro plástico de anillos Pall de nylon acrodisc de 0.2 $\mu \mathrm{m}$ para posteriormente ser analizadas en el equipo HPLC.

Para el análisis de temperatura se utilizó una concentración inicial de $0.1 \mathrm{mM}$ de BPA y $100 \mathrm{mg} / \mathrm{L}$ de $\mathrm{TiO}_{2}$ y se evaluó la reacción con 4 temperaturas, medidas según el promedio entre la entrada y salida del reactor: $22.52 \pm 2.61,35.13 \pm 2.71,46.81 \pm 3.45,58.54 \pm 4.06$ ${ }^{\circ} \mathrm{C}$. Se utilizó el mismo montaje pero se varió la temperatura en la plancha de agitación. Se empleo una termocupla de mercurio con precisión de $\pm 0.1^{\circ} \mathrm{C}$ para registrar la temperatura cada $30 \mathrm{~min}$ en cuatro puntos del circuito: en la entrada y salida del reactor, en el interior del Beaker y en la corriente de reciclo.

\section{Procedimiento experimental $\mathrm{TiO}_{2}$ inmovilizado}

Se desarrollaron películas de tres materiales; acrílico, acetato y vidrio, con el fin de encontrar la mejor alternativa para fijar $\mathrm{TiO}_{2}$. Se preparó una solución concentrada de $5 \mathrm{~g} / \mathrm{L}$ de $\mathrm{TiO}_{2}$ en agua, la cual fue depositada sobre cada superficie. En cuanto al acrílico y al acetato, se dejó secar la solución a temperatura ambiente, con el fin de evaporar el agua de la solución. Mientras que el vidrio fue activado con incrementos de $50{ }^{\circ} \mathrm{C}$ de temperatura hasta llegar a $400{ }^{\circ} \mathrm{C}$ durante $1 \mathrm{~h}$, empleando una mufla 47900 Barnstead.

Las películas se introdujeron al reactor foto-catalítico, ubicándolas en el espacio libre entre la pared del tubo pirex y la lámpara UV. De tal forma que la solución acuosa de $0.1 \mathrm{mM}$ de BPA fluyera a lo largo de reactor y las láminas tuvieran contacto con la luz. Cada prueba fue de $4 \mathrm{~h}$ tomando muestras cada $30 \mathrm{~min}$.

La cuantificación de la concentración de BPA se realizó con el equipo HPLC al igual que con el catalizador en suspensión pero además se utilizó una balanza con una precisión de $\pm 0.01 \mathrm{mg}$ para cuantificar la cantidad de $\mathrm{TiO}_{2}$ mediante la diferencia de peso. Estos datos se tomaron $24 \mathrm{~h}$ después de finalizada la degradación para que el agua se evaporara.

\section{Análisis HPLC}

Se utilizó HPLC (High Performance Liquid Chromatography) para cuantificar la concentración de Bisfenol A. Las condiciones empleadas en este análisis cuantitativo de BPA fueron: Una fase móvil de $20 \mathrm{mM}$ de fosfato de sodio como solución buffer ( $\mathrm{pH} 7$ ) y acetonitrilo en proporción 65\%:35\% y un flujo volumétrico de $1 \mathrm{~mL} /$ min. Se utilizó una columna analítica de arreglo de diodos con una longitud de $250 \mathrm{~mm}$ (Allsphere ODS 2-5 $\mu$ ), +0.35/0,55 V, temperatura $20 \pm 3{ }^{\circ} \mathrm{C}$. El método empleado fué isocrático, inyectando 10 $\mu \mathrm{L}$ y el tiempo de corrida fue de 30 min por muestra.

\section{RESULTADOS Y DISCUSION}

\section{Implementación de los métodos de análisis}

En el cromatograma HPLC de una solución de $0.1 \mathrm{mM}$ de BPA, se obtuvo el tiempo de retención de $18.63 \mathrm{~min}$ el cual coincide con el reportado en la literatura. ${ }^{11}$ Esta señal fue corroborada con el análisis de la solución estándar. El espectro UV del BPA presentó un máximo de absorción a $275 \mathrm{~nm}$ correspondiente al reportado en la literatura. ${ }^{11}$

La curva de calibración fue construida a partir de las concentraciones patrón de BPA en el rango 0.007 a $0.2 \mathrm{mM}$, con un límite de detección de $0.004 \mathrm{mM}$. Esta curva reportó un coeficiente de correlación de 0.9995. La desviación estándar porcentual de los datos posee valores relativamente bajos (1.91-13.16\%) indicando que los resultados son repetibles y reproducibles en el rango mencionado. 


\section{Fotodegradación catalítica de $\mathrm{BPA}$ empleando $\mathrm{TiO}_{2}$ en suspensión}

Se analizaron dos variables: la cantidad de dióxido de titanio y la temperatura de reacción.

\section{Efecto de la cantidad de catalizador $\mathrm{TiO}_{2}$}

La Figura 1 presenta el efecto de la cantidad de $\mathrm{TiO}_{2}$ en la cinética de fotodegradación de una solución acuosa de $0.1 \mathrm{mM}$ BPA. En esta figura se observa que la foto-catálisis es favorecida por el incremento de la cantidad de $\mathrm{TiO}_{2}$ en el rango entre $0-100 \mathrm{mg} / \mathrm{L}$. No obstante después de este rango empleando cantidades superiores, se observa una disminución en la eficiencia de remoción de contaminante.

Estos perfiles de concentración respecto al tiempo de degradación pueden ser correlacionados mediante la velocidad de reacción de foto-catálisis empleando el método integral para una reacción de primer orden, como se muestra a continuación:

$r=-\frac{d C a}{d t}=k_{a p} C a$

donde $\mathrm{k}_{\text {ap }}$ es la constante aparente, $\mathrm{Ca}=$ concentración de $\mathrm{BPA}, \mathrm{r}=$ velocidad de reacción.

Tomando como límites de la integral $\mathrm{Ca}=\mathrm{Cao}$ en $\mathrm{t}=0$, se obtiene la relación:

$\ln \frac{C a o}{C a}=k_{a p} t$

Esta linealización permite aproximar la foto-catálisis del BPA a un modelo de reacción de pseudo primer orden ya que existe un buen ajuste de los datos según el rango del coeficiente de correlación 0.80.99. A partir de las linealizaciones de la ecuación de velocidad de reacción para $0.01 ; 0.05 ; 0.1 \mathrm{mM}$ de BPA variando la concentración de catalizador $0 ; 100 ; 250 ; 500 \mathrm{mg} / \mathrm{L}$ de $\mathrm{TiO}_{2}$, se determinó directamente la constante de velocidad de reacción aparente $\left(\mathrm{K}_{\text {ap }}\right)$ que es equivalente a la pendiente de cada una de las regresiones lineales.

La Figura 1 presenta la evolución de la constante aparente de reacción $\left(\mathrm{K}_{\mathrm{ap}}\right)$ en función de la cantidad de catalizador. Se observa que $\mathrm{K}_{\text {ap }}$ posee valores mayores para una concentración de Bisfenol A menor. Esto indica que la reacción de fotodegradación ocurre más rápido para concentraciones bajas debido a que existe menos competencia por los sitios activos disponibles en una cantidad de catalizador determinada. ${ }^{22} \mathrm{Se}$ observa que la tendencia de $\mathrm{K}_{\text {ap }}$ respecto a la concentración de $\mathrm{TiO}_{2}$ es similar para las tres concentraciones de BPA. Inicialmente la constante aparente posee un comportamiento ascendente cuando se utilizan cantidades de $\mathrm{TiO}_{2}$ menores o iguales a $100 \mathrm{mg} / \mathrm{L}$ y descendiente para concentraciones mayores. La mayor $\mathrm{K}_{\text {ap }}\left(0.0154 \mathrm{~min}^{-1}\right)$ se obtiene cuando la concentración inicial de BPA es $0.01 \mathrm{mM}$ y la cantidad de $\mathrm{TiO}_{2}$ es $100 \mathrm{mg} / \mathrm{L}$.

La disminución de la constante aparente de reacción al incrementar la cantidad de $\mathrm{TiO}_{2}$ a una cantidad superior a $100 \mathrm{mg} / \mathrm{L}$, puede ser explicado a partir de los siguientes tres fenómenos.

\section{Formación de intermediarios en el proceso foto-catalítico}

Según la literatura, la foto-catálisis heterogénea con $\mathrm{TiO}_{2}$ puede descomponer la molécula de BPA generando productos intermediarios de degradación, hasta llegar a una posible mineralización o formación de $\mathrm{CO}_{2} \cdot{ }^{5}$ Estudios presentan posibles vías de degradación del BPA, los cuales postulan que algunos de los productos intermediarios pueden ser 4-isopropilfenol, 4- hidroxibenzaldehido, hidroquinona, hidroxiacetofenona, o algunos ácidos carboxílicos como: acido acéti-

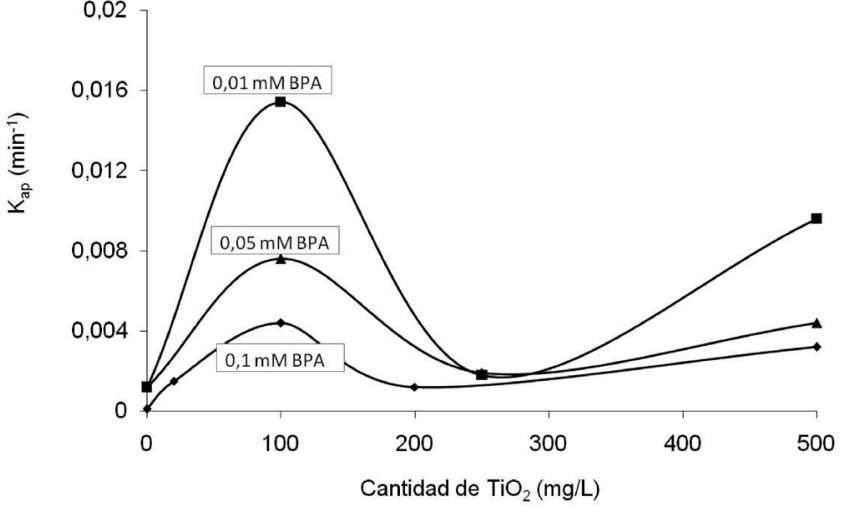

Figura 1. Evolución de la constante aparente de reacción $\left(K_{a p}\right)$ en función de la cantidad de $\mathrm{TiO}_{2}$ utilizado en los experimentos de degradación fotocatalítica de BPA con diferentes concentraciones iníciales

co, acido fórmico. ${ }^{5,6} \mathrm{La}$ formación de estos productos intermediarios puede limitar o inhibir la reacción de foto-catálisis, generando una disminución en la constante aparente de reacción después de $100 \mathrm{mg} / \mathrm{L}$ de $\mathrm{TiO}_{2}$ (Figura 1). Esto implica una disminución en la eficiencia de remoción del contaminante, debido a que estas nuevas moléculas generan una competencia por los sitios activos del catalizador y hacen que la reacción sea más lenta.

\section{Efecto apantallamiento}

La literatura nombra un efecto de apantallamiento cuando la concentración de $\mathrm{TiO}_{2}$ es muy alta. Este fenómeno se ve reflejado en la disminución de la velocidad de reacción debido a la excesiva opacidad de la disolución disminuyendo el rendimiento del proceso foto-catalítico. ${ }^{21}$ Una alta cantidad de partículas de $\mathrm{TiO}_{2}$ en suspensión contribuye a la formación de una pantalla que impide que los fotones de luz alcancen a las partículas de $\mathrm{TiO}_{2}$ y al contaminante generando una disminución de la constante aparente $\left(\mathrm{K}_{\mathrm{ap}}\right)$.

\section{Adsorción de BPA en la superficie del catalizador TiO}

Se observó una remoción correspondiente al $7.5 \%$ de BPA en ausencia de luz UV, este fenómeno puede ser atribuido a fenómenos de adsorción.

Según la literatura y estudios recientes, para describir el fenómeno de adsorción en foto-catálisis heterogénea, el modelo más empleado es el de Langmuir - Hinshelwood, el cual relaciona la velocidad de reacción y la concentración inicial de contaminante. Este modelo asume que la adsorción ocurre exclusivamente en sitios específicos localizados sobre la superficie del adsorbente (monocapa), donde solamente una molécula de adsorbato se adsorbe sobre cada sitio. La expresión que relaciona la adsorción según los supuestos de Langmuir es:

$q=\frac{q_{m} K_{s} C_{o}}{1+K_{s} C_{o}}$

donde $\mathrm{C}_{\mathrm{o}}=$ concentración del soluto en equilibrio $(\mathrm{mg} / \mathrm{L}), \mathrm{q}=$ masa de soluto adsorbido por unidad de masa de adsorbente $(\mathrm{mg} / \mathrm{g}), \mathrm{q}_{\mathrm{m}}=$ máxima masa de soluto que se adsorbe sobre el adsorbente $(\mathrm{mg} / \mathrm{g})$, $\mathrm{K}_{\mathrm{s}}=$ constante de equilibrio de Langmuir relacionada con el calor de adsorción (L/mg).

Teniendo en cuenta la expresión de Langmuir- Hinshelwood y que la reacción es de primer orden, la velocidad de reacción puede expresarse de la siguiente forma: ${ }^{12}$

$r=k \frac{K_{s} C}{1+K_{s} C_{o}}=k_{a p} C$ 
donde k es una constante que relaciona la adsorción en la superficie para una reacción de primer orden, particularmente hace referencia a los huecos generados por el desprendimiento de los radicales ${ }^{\circ} \mathrm{OH}$ y $\mathrm{k}_{\text {ap }}$ corresponde a la constante aparente de la velocidad de reacción. La linealización de esta expresión se nuestra a continuación: ${ }^{12}$

$\frac{1}{k_{a p}}=\frac{1}{k K_{s}}+\frac{C o}{k}$

Esta expresión permite relacionar adsorción y la reacción en superficie con la concentración inicial de BPA. La Figura 2 muestra la linealización de la Ecuación 8 donde se muestra la tendencia de la inversa de la constante aparente vs la concentración inicial de BPA para diferentes concentraciones de dióxido de titanio, permitiendo obtener los valores de las constantes $k, K_{s}$. El ajuste de los datos fue bueno con un rango de coeficientes de correlación entre 0.73-0.99.

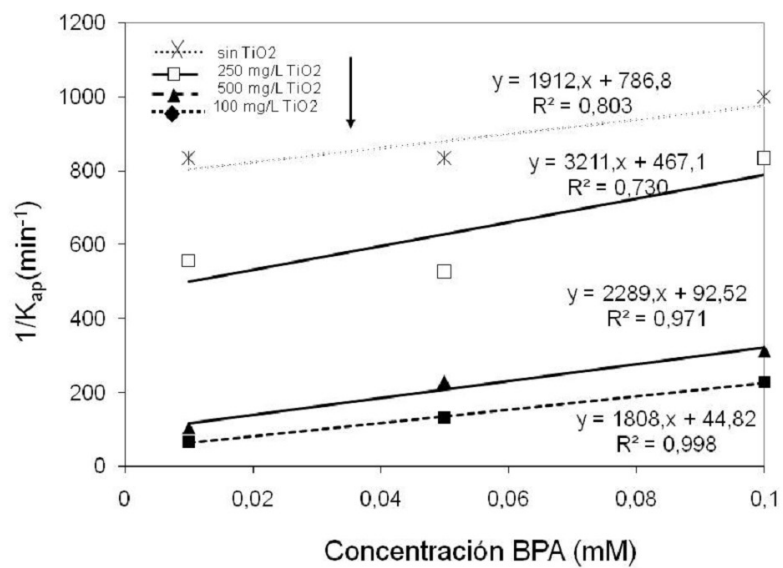

Figura 2. Evolución de la relación 1/Kap en función de la concentración Bisfenol A y de la cantidad de TiO2 utilizado en la fotodegradación catalítica de BPA

Los valores de las constantes de reacción en la superficie $k$ encontrados a partir de las regresiones lineales se relacionan con la concentración de $\mathrm{TiO}_{2}$ se muestran en la Figura 3. Se puede observar que el comportamiento de $\mathrm{k}$ posee una tendencia similar al de $\mathrm{k}_{\mathrm{ap}}$. Sin embargo los valores de la $\mathrm{k}_{\mathrm{ap}}$ se encuentran en un rango superior entre 0.001 $0.0154 \mathrm{~min}^{-1}$, mientras que la k están entre 0.00031-0.0012 $\mathrm{min}^{-1}$.

En la Figura 4 se relaciona la constante de equilibrio de adsorción $K_{c}$ con respecto a la concentración de $\mathrm{TiO}_{2}$, se observa que $K_{c}$ posee una tendencia descendente respecto a la concentración de catalizador. En ausencia de $\mathrm{TiO}_{2}$ la constante inicia en un valor de $0.41 \mathrm{~L} \mathrm{mmol}^{-1}$ y posteriormente desciende hasta $0.08 \mathrm{~L} \mathrm{mmol}^{-1}$ cuando se emplea $100 \mathrm{mg} / \mathrm{L} \mathrm{TiO}_{2}$, este valor puede ser considerado como un mínimo local ya que con $250 \mathrm{mg} / \mathrm{L}$ de $\mathrm{TiO}_{2}$ el valor de la constante $K_{c}$ incrementa hasta un valor de $0.16 \mathrm{~L} \mathrm{mmol}^{-1} \mathrm{y}$ al continuar aumentando la concentración de catalizador se observa que el valor de esta variable continua descendiendo. Este comportamiento muestra que existe un mecanismo de adsorción en la reacción foto-catalítica, cuando se utiliza $100 \mathrm{mg} / \mathrm{L}$ de $\mathrm{TiO}_{2}$ la adsorción es lenta respecto a las demás concentraciones de catalizador, mientras que al manejar $250 \mathrm{mg} / \mathrm{L}$ de $\mathrm{TiO}_{2}$ la adsorción es rápida.

Según las Figuras 3 y 4 , se observa que los valores de $K_{c}$ son notablemente mayores que los valores de $k$. El rango de $K_{c}$ se encuentra entre 0.05-0.41 $\mathrm{min}^{-1}$, mientras que los valores de $k$ están entre 0.0003-0.0012 $\min ^{-1}$. Estos resultados indican que la reacción de adsorción es más rápida que la reacción en la superficie, permitiendo predecir que la etapa limitante de la degradación de BPA es la reacción en superficie.

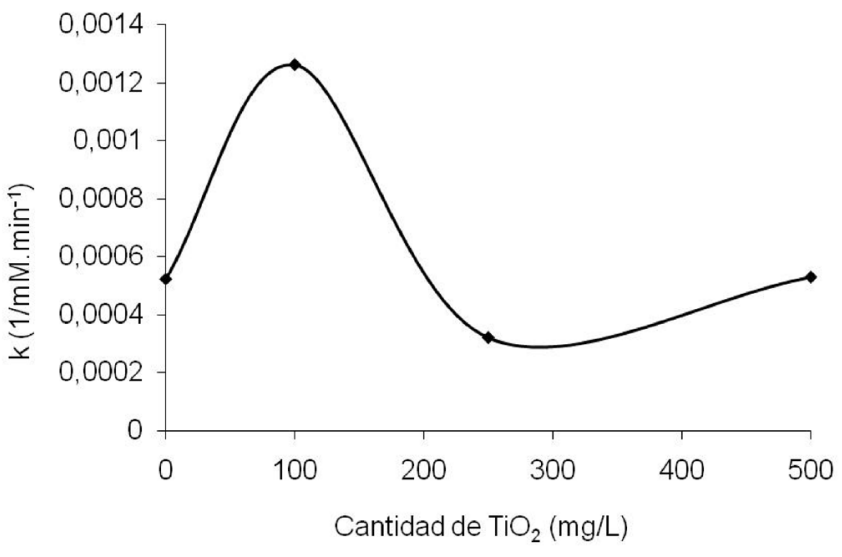

Figura 3. Evolución de la constante de superficie ( $k$ ) en función de la cantidad de $\mathrm{TiO}_{2}$ utilizado en la fotodegradación de BPA

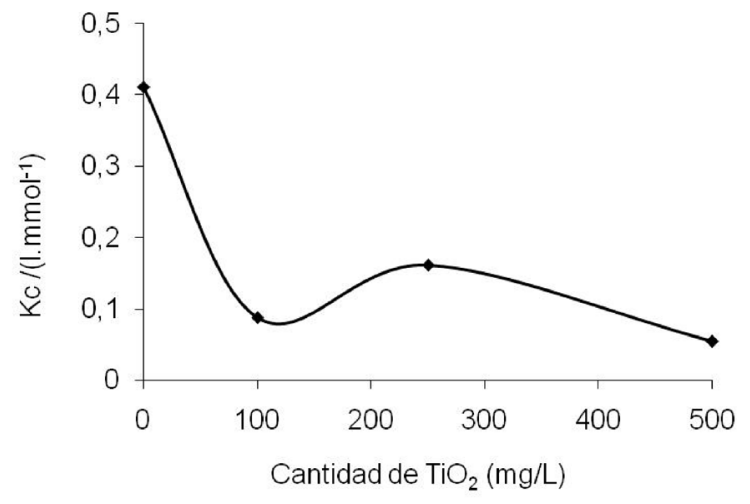

Figura 4. Evolución de la constante de equilibrio de absorción (Kc) en función de la cantidad de $\mathrm{TiO}_{2}$

Los resultados muestran que con $100 \mathrm{mg} / \mathrm{L}$ de $\mathrm{TiO}_{2}$ la reacción ocurre más rápido que con concentraciones mayores de catalizador donde se evidencia una disminución en la rapidez de reacción ya que la constante de la reacción en la superficie $k$ tiende a aumentar y la constante de equilibrio de adsorción $K_{c}$ tiende a disminuir, mientras que para concentraciones mayores ocurre lo inverso, esto indica que la reacción en la superficie se vuelve más lenta al incrementar la concentración de catalizador, lo cual puede ser causa de una disminución en el numero de sitios activos en el catalizador donde las moléculas de contaminante tienen que competir para ocupar un sitio disponible donde llevar a cabo la reacción.

\section{Efecto de la temperatura}

La velocidad de reacción es generalmente función de la concentración de reactivos y de la temperatura. La relación entre la constante de reacción y la temperatura se expresa mediante la Ecuación de Arrhenius: ${ }^{13}$

$k_{a p}=A \cdot e^{\frac{-E a}{R T}}$

donde $\mathrm{k}_{\mathrm{ap}}=$ constante de velocidad de reacción, $\mathrm{E}_{\mathrm{a}}=$ energía de activación, $\mathrm{R}=$ constante de los gases, $\mathrm{T}=$ temperatura a la que ocurre la reacción, $A=$ factor de frecuencia.

Para poder determinar la energía de activación experimental a partir de la Ecuación de Arrhenius, se evaluó la reacción con 4 temperaturas diferentes $(22.54 \pm 2.61,35.17 \pm 2.71,46.82 \pm 3.45$ 
y $58.51 \pm 4.06^{\circ} \mathrm{C}$ ) las cuales fueron medidas en la entrada y salida de la lámpara.

Se calculó la constante de reacción aparente $\left(K_{a p}\right)$ en una reacción de primer orden para cada una de las temperaturas en las que se evaluó la degradación de BPA. La temperatura que generó una menor constante de reacción $\left(K_{a p}\right)$ fue la correspondiente a $58.51 \pm 4.06{ }^{\circ} \mathrm{C}$, esto se debe a que según la expresión de Arrhenius a que una mayor temperatura se genera un incremento, ${ }^{13}$ disminuyendo el tiempo de reacción de tal forma que los reactantes son convertidos rápidamente en la superficie del catalizador.

La Figura 5 muestra la linealización de Arrhenius donde la pendiente de la recta corresponde a $-E_{a p} / R$ y el corte con el eje $Y$ corresponde a $\ln A$.

$$
\ln k_{a p}=\frac{-E}{R}\left(\frac{1}{T}\right)+\ln A
$$

El valor numérico de la energía de activación $\left(\mathrm{E}_{\mathrm{ap}}\right)$ para un rango de temperatura entre $20-60^{\circ} \mathrm{C}$ es de $14.54 \mathrm{KJ} / \mathrm{mol}$ y el factor de forma (A) es igual 1.47 el cual hace referencia al número de colisiones o a la concentración de sitios catalíticos. El valor obtenido de $\mathrm{E}_{\text {ap }}$ se encuentra dentro del rango de los valores numéricos reportados en la literatura de 8.8-18.1 KJ/mol para compuestos orgánicos expuestos a foto-degradación catalítica con $\mathrm{TiO}_{2}$ en suspensión para una temperatura de operación entre 10 y $60{ }^{\circ} \mathrm{C}$.

\section{Fotodegradación catalítica de BPA empleando $\mathrm{TiO}_{2}$ inmovilizado}

En un proceso catalítico, el número de moléculas de reactante que son convertidas a producto en un periodo de tiempo determinado está relacionado directamente con el número de sitios catalíticos disponibles. Es por esto que se busca maximizar el número de sitios activos disponibles fijando el catalizador en soportes que posibiliten una mayor cantidad de sitios donde ocurre la reacción y la formación de producto. Esto genera una necesidad de desarrollar catalizadores soportados que posibiliten una alta eficiencia de remoción de contaminantes para poder ser empleados a nivel industrial y de esta manera reducir los costos de recuperación y de separación con procesos como filtración.

En la Tabla 1 se muestra una comparación entre los sustratos empleados en la fijación de $\mathrm{TiO}_{2}$, para evaluar el proceso de foto degradación de una solución de $0.1 \mathrm{mM}$ de BPA. Para determinar la cantidad de $\mathrm{TiO}_{2}$ presente en cada sustrato, se empleo una balanza y se comparo el peso antes y después de la fijación.

De acuerdo a los resultados de la Tabla 1, se puede observar que el sustrato que genera una mayor remoción de BPA es el acetato, sin embargo se desprendió el $95 \%$ del $\mathrm{TiO}_{2}$ inmovilizado por lo tanto

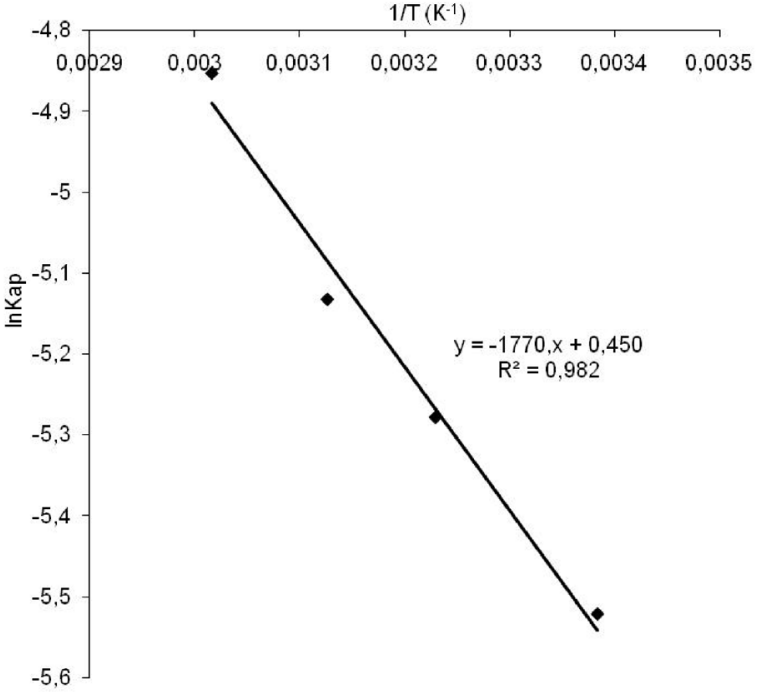

Figura 5. Efecto de la temperatura sobre la constante de velocidad aparente de la fotodegradación heterogénea de BPA. Linealización de la ecuación de Arrhenius

estos resultados son equivalentes a la foto degradación empleando el catalizador en suspensión. Debido a esta deficiencia en la fijación sobre acetato, se empleo laca comercial con el fin de mejorar adherencia reduciendo el desprendimiento a el $1.5 \%$ del $\mathrm{TiO}_{2}$ inicial, sin embargo se logró una remoción de BPA del 23.9\%. Estos resultados indican que la luz genera radicales hidroxilo sin embargo la barrera que produce la laca hace que la desorción de los productos desde la superficie del catalizador sea más lenta. Además se notó una variación en el color inicial de la lámina, pasando de blanco a amarillo. Este cambio en el color puede ser atribuido a la oxidación de un componente debido a la presencia de $\mathrm{TiO}_{2}$ o a que algún aditivo refracte la luz en la laca generando una coloración en la película. ${ }^{14}$

Respecto al acrílico, se logró remover $25.67 \%$ del contaminante, sin embargo no fué posible cuantificar la cantidad de catalizador que se desprendió de la lamina durante la prueba, debido a que el peso final del las laminas después de $24 \mathrm{~h}$ fue superior al inicial. Esto puede ser causado a fenómenos de adsorción en el acrílico.

El mejor resultado obtenido fue empleando vidrio como sustrato. Se alcanzó una remoción de $47.65 \%$ de BPA y un desprendimiento del $52.01 \%$ de $\mathrm{TiO}_{2}$ del soporte. Estas laminas fueron reutilizadas logrando un $21.15 \%$ de remoción y un $62.02 \%$ de desprendimiento (Figura 6). Una de las características favorables de este material es que uno de sus componentes es el hierro el cual tiene la propiedad de adsorber radiación UV, esto hace que sea más sencillo para el catalizador inmovilizado captar la luz y producir radiales ${ }^{\circ} \mathrm{OH}$.

Tabla 1. Fotodegradación de $0.1 \mathrm{mM}$ BPA con $\mathrm{TiO}_{2}$ fijo

\begin{tabular}{|c|c|c|c|c|c|}
\hline Sustrato & $\mathrm{DL}(\mathrm{cm})$ & $\mathrm{TiO}_{2}$ fijo (mg) & VL/Vf (\%) & R.BPA (\%) & D. $\mathrm{TiO}_{2}(\%)$ \\
\hline Acetato & 7.5 X11 0.5 & 102,09 & 12,95 & 60 & 95 \\
\hline Acetato + Laca & $7.5 \times 110.5$ & 114,5 & 12,95 & 23,9 & 1,5 \\
\hline Acrílico & $1.5 \times 5.0 \times 0.7$ & 177,4 & 21,15 & 25,67 & Adsorción \\
\hline Vidrio $1 \mathrm{vez}$ & $1.5 \times 7.5 \times 0.3$ & 151,4 & 19,42 & 47,65 & 52 \\
\hline Vidrio $2 \mathrm{vez}$ & $1.5 \times 7.5 \times 0.3$ & 68 & 19,42 & 21,15 & 62 \\
\hline
\end{tabular}

$\mathrm{DL}=$ dimensiones de las laminas, $\mathrm{VL} / \mathrm{Vf}=\%$ volumen ocupado por las laminas respecto al volumen de flujo del reactor, $\mathrm{R} . \mathrm{BPA}=\%$ remoción de $\mathrm{BPA}, \mathrm{D} . \mathrm{TiO}_{2} \%=\mathrm{TiO} 2$ que se desprende del soporte. 


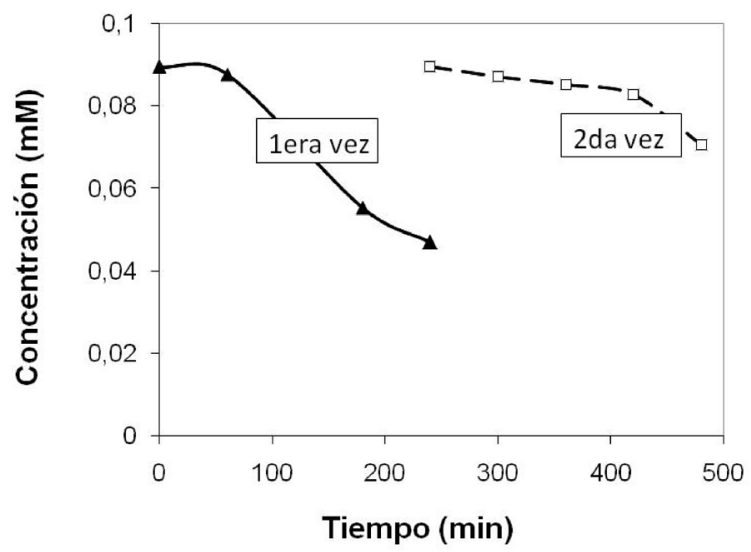

Figura 6. Evaluación de la estabilidad del fotocatalizador $\mathrm{TiO}_{2}$ inmovilizado sobre láminas de vidrio en la fotodegradación heterogénea de BPA

De acuerdo a los resultados de la Figura 6, se observa que la remoción de contaminante con la reutilización del catalizador soportado ( 2 vez) es menor que la prueba inicial ( $1 \mathrm{vez}$ ), esto se debe a que un gran porcentaje del dióxido de titanio se desprendió de la lámina de vidrio por diversos factores como rozamiento con las paredes del reactor, la velocidad de flujo de la solución o por deficiencia en la fijación del $\mathrm{TiO}_{2}$. Esto implica que para incrementar la eficiencia de remoción, es necesario el uso de un aglutinante que permita la fijación del catalizador en el soporte.

Comparación entre el dióxido de titanio en suspensión e inmovilizado

Teniendo en cuenta que el montaje utilizado para los dos estados del catalizador es el mismo, se debe considerar que existen algunas condiciones que varían y modifican los resultados considerablemente.

Se encontró que la eficiencia foto-catalítica con el catalizador soportado en vidrio, acetato y acrílico es menor que la eficiencia empleando el catalizador en suspensión. Sin embargo la mayor remoción de $0.1 \mathrm{mM}$ de BPA con el catalizador soportado es cuando se emplea el vidrio como sustrato removiendo el $47.1 \%$ de BPA en $240 \mathrm{~min}$, mientras que empleando el $\mathrm{TiO}_{2}$ en suspensión la mayor remoción alcanzada fue de $65 \%$ de BPA. Esto puede explicarse debido a la variación de las condiciones de cada una de las pruebas. El catalizador en suspensión circula en todo el circuito del montaje, mientras que cuando se tiene el catalizador inmovilizado únicamente se utiliza el 5.1\% del volumen del reactor, esto implica una reducción en el tiempo de residencia y en el área de contacto entre el dióxido de titanio y el contaminante.

Con respecto a la transferencia de masa, el soporte limita en el transporte de materia entre los reactantes y los productos, debido a que se pueden generar barreras que obstaculizan el flujo de electrones. Además, la dispersión del dióxido de titanio puede causar aglomeración y acumulación en determinados lugares del soporte, lo cual impide que partículas de catalizador se activen con la luz y logren formar radicales ${ }^{\circ} \mathrm{OH}$. Esto hace que la superficie de $\mathrm{TiO}_{2}$ activada sea menor con respecto a la que se genera en suspensión. Sin embargo, aunque los soportes implican algunas desventajas, en el proceso de foto-catálisis industrial resulta más efectivo emplear el catalizador inmovilizado. Es por esto que actualmente gran parte de estudios intentan implementar procesos para reducir las deficiencias de los catalizadores fijos en diversas superficies debido a que se evita la separación y recuperación del catalizador posterior al tratamiento, a que no ocurre el efecto de apantallamiento impidiendo que la luz llegue a todos los sitios activos, ni tampoco ocurre acumulación de catalizador. Estas condiciones implican una reducción en los costos de operación debido a que se requiere de un número menor de operaciones y procesos para obtener el producto deseado.

\section{CONCLUSIONES}

Se estandarizó la técnica de análisis de BPA en agua, por medio de cromatografía liquida (HPLC), obteniendo resultados fiables hasta 0.0034-0.004 mM de BPA.

Se evaluó el efecto de la cantidad de $\mathrm{TiO}_{2}$ en la foto-degradación de BPA, encontrando que con $100 \mathrm{mg} / \mathrm{L}$ de $\mathrm{TiO}_{2}$ en una solución $0.05 \mathrm{mM}$ BPA, se logra una mayor remoción a los $240 \mathrm{~min}$ del $83 \%$,

Se encontró que existe una cantidad adecuada de catalizador debido a que algunos factores como: formación de productos intermediarios de degradación, efecto apantallamiento, adsorción y disminución de sitios activos disponibles en la superficie del $\mathrm{TiO}_{2}$, inhiben la reacción causando una disminución en la eficiencia de la foto-catálisis.

Se encontró que para una concentración de BPA mayor manteniendo fijo la cantidad de $\mathrm{TiO}_{2}$, la reacción de foto degradación es más lenta. Esto se debe a que se requiere de un mayor número de sitios activos para degradar una concentración superior de contaminante.

Se determinó que a una mayor temperatura promedio en el reactor correspondiente a $58.51 \pm 4.06^{\circ} \mathrm{C}$ la reacción se lleva a cabo más rápido. Esto se debe a la relación exponencial entre la temperatura de reacción y la constante de reacción expresadas en la ecuación de Arrhenius.

Se calculó la energía de activación y el coeficiente de forma, encontrando que los valores $14.543 \mathrm{KJ} / \mathrm{mol}$ y 1.47 , se encuentran dentro del rango de los valores reportados en la literatura.

Se probaron tres materiales como soporte del dióxido de titanio, resultado el vidrio como mejor sustrato en cuanto a fijación y remoción de BPA.

\section{AGRADECIMIENTOS}

Los autores manifiestan su agradecimiento a la Facultad de Ciencias de la Universidad de los Andes y su Fondo Semilla, por el apoyo económico.

\section{REFERENCIAS}

1. Andrade, A.; Pacheco, A.; Nóbrega da Cunh, A.; Biomedica 2006, 17,146 .

2. Montezchua, E.; Zamarripa, H.; Leyva, E.; Rev. Int. Contam. Ambient. 2003, 19, 117 .

3. Colborn, T.; Dumanoski, D.; Peterson, J.; Our Stolen Future, Penguin Books: New York, 1996.

4. Dumanoski y Pete Myers; Ecoespaña y Gaia-Proyecto 2050, Madrid. 1997.

5. Watanabe, N.; Horikoshi, S.; Kawabe, H.; Sugie, Y.; Zhao, J.; Hidaka, H.; Chemosphere 2002, 52, 851.

6. Gwynne, L.; Bisphenol A: A Known Endocrine Disruptor, A WWF European Toxics Programme Report, April 2000.

7. Hun Kang, J.; Kondo, F.; Katayama, Y.; Toxicology 2006, 226, 79.

8. United States Environmental Protection Agency; Advanced Photochemical Oxidation Processes, EPA, 1998

9. Sarria, V.; Parra, S.; Adler, N.; Péringer, P.; Pulgarin, C.; Catal. Today 2002, 76, 301

10. Chen, J.; Rosenfeldt, E. J.; Kullman, S. W.; Hinton, D. E.; Linden, K. G.; Sci. Total Environ. 2006, 376,18.

11. Fukahori, S.; Ichiura, H.; Kitaoka, T.; Tanaka, H.; Appl. Catal., B 2003, $46,453$.

12. Youji, L.; Xiaodong, L.; Junwen, L.; Jing, Y.; Water Res. 2006, 40, 1119.

13. Smith, J. M.; Chemical Engineering Kinetics, $3^{\text {rd }}$ ed., McGraw Hill: New York, 1981.

14. Cheheb, Z.; Albouchi, F.; Nasrallah, S. B.; J. Quant. Spectrosc. Radiat. Transfer. 2007, 109, 620. 